\title{
On Some Subclasses of $m$-fold Symmetric Bi-univalent Functions associated with the Sakaguchi Type Functions
}

\author{
Ismaila O. Ibrahim ${ }^{1}$, Timilehin G. Shaba ${ }^{2, *}$ and \\ Amol B. Patil ${ }^{3}$ \\ ${ }^{1}$ Department of Mathematical Science, University of Maiduguri, Nigeria \\ e-mail: ibrahimismailaomeiza@gmail.com \\ 2 Department of Mathematics, University of Ilorin, P. M. B. 1515, Ilorin, Nigeria \\ e-mail: shabatimilehin@gmail.com \\ ${ }^{3}$ Department of First Year Engineering, AISSMS's College of Engineering, Pune-411001, India \\ e-mail: amol223patil@yahoo.co.in
}

\begin{abstract}
In the present investigation, we introduce the subclasses $\Lambda_{\Sigma_{m}}^{\widehat{\Lambda}}(\sigma, \phi, v)$ and $\Lambda_{\Sigma_{m}}(\sigma, \gamma, v)$ of $m$-fold symmetric bi-univalent function class $\Sigma_{m}$, which are associated with the Sakaguchi type of functions and defined in the open unit disk. Further, we obtain estimates on the initial coefficients $b_{m+1}$ and $b_{2 m+1}$ for the functions of these subclasses and find out connections with some of the familiar classes.
\end{abstract}

\section{Introduction}

Let $\mathcal{N}$ and $\mathcal{C}$ represent the sets of natural numbers and complex numbers respectively and $\mathcal{A}$ be the family of analytic functions that are defined in $\mathcal{U}=\{z \in \mathcal{C}:|z|<1\}$ and have the series expansion

$$
f(z)=z+\sum_{k=2}^{\infty} a_{k} z^{k} \quad(z \in \mathcal{U}, k \in \mathcal{N})
$$

Received: September 10, 2021; Accepted: October 13, 2021

2010 Mathematics Subject Classification: 30C55, 30C45.

Keywords and phrases: univalent function, bi-univalent function, $m$-fold symmetric bi-univalent function, Sakaguchi type function.

${ }^{*}$ Corresponding author 
A function $f \in \mathcal{A}$ be a member of the family $\mathcal{S}$ if it is univalent in $\mathcal{U}$. Applying Koebe 1-quarter theorem (see [5]) we can ensure an inverse to each $f \in \mathcal{S}$ defined as

$$
f^{-1}(f(z))=z \quad \text { for } \quad z \in \mathbb{U}
$$

and

$$
f\left(f^{-1}(w)\right)=w \quad \text { for } \quad|w|<r_{0}(f) \quad \text { and } \quad r_{0}(f) \geq 1 / 4,
$$

with series expansion

$$
f^{-1}(w)=w+\left(-a_{2}\right) w^{2}+\left(2 a_{2}^{2}-a_{3}\right) w^{3}+\left(5 a_{2} a_{3}-5 a_{2}^{3}-a_{4}\right) w^{4}+\cdots .
$$

Let $\Sigma=\left\{f: \mathcal{U} \rightarrow \mathcal{C}\right.$ such that $\left.f, f^{-1} \in \mathcal{S}\right\}$ be the family of bi-univalent functions. This family $\Sigma$ was introduced in the year 1967 by Lewin [10] along with the result $\left|a_{2}\right|_{f \in \Sigma}<1.51$. Afterverse, Brannan and Clunie [3] stated that $\left|a_{2}\right|_{f \in \Sigma} \leq \sqrt{2}$. Further, Goodman [7] thought that $\left|a_{n}\right|_{f \in \Sigma} \leq 1$ may be true for every $n \in \mathbb{N}$. However, Netanyahu [12] ensured that $\max _{f \in \Sigma}\left|a_{2}\right|=4 / 3$. But, then Styer and Wright [29] guaranteed the existence of $f \in \Sigma$ for which $\left|a_{2}\right|>4 / 3$.

Indeed, the study of the class $\Sigma$ has been accelerated greatly due to the fundamental work of Srivastava et al. [27]. After that many researchers ([1], [4], [6], 8], [13], [17], [19], 24], 20], [21], [23], [14] etc.) found coefficient estimates for functions in several subclasses of $\Sigma$. But still the coefficient bound problem of $\left|a_{n}\right|,(n=3,4,5, \cdots)$ for the functions in $\Sigma$ is open.

A function $p(z)$ given by

$$
p(z)=z+\sum_{k=1}^{\infty} b_{(m k+1)} z^{(m k+1)} \quad(z \in \mathcal{U}, m \in \mathcal{N})
$$

is known as the $m$-fold symmetric function (see [9], [16]). Moreover, a function $u(z)$ of the form

$$
u(z)=\left(f\left(z^{m}\right)\right)^{1 / m} \quad(f \in \mathcal{S}, z \in \mathcal{U}, m \in \mathcal{N})
$$

is univalent and maps $\mathcal{U}$ into a $m$-fold symmetric region. Let $\mathcal{S}_{m}$ be the class of univalent and $m$-fold symmetric functions in $\mathcal{U}$, which are of the form 1.3. 
Observe that for $m=1$, these functions become members of the class $\mathcal{S}$ and are known as symmetric univalent functions.

For each $m \in \mathcal{N}$, every $f \in \Sigma$ produces a $m$-fold symmetric bi-univalent function. For a function $p$ of the form (1.3), an univalent continuation of $p^{-1}$ to $\mathcal{U}$ is given by (see Srivastava et al. [28]) the series expansion

$$
\begin{aligned}
q(w)= & w-b_{m+1} w^{m+1}+\left[(m+1) b_{m+1}^{2}-b_{2 m+1}\right] w^{2 m+1}- \\
& {\left[\frac{1}{2}(m+1)(3 m+2) b_{m+1}^{3}-(3 m+2) b_{m+1} b_{2 m+1}+b_{3 m+1}\right] w^{3 m+1} } \\
& +\cdots
\end{aligned}
$$

See that for $m=1$, it reduces to the equation 1.2 . Hence, we can generalize the class $\Sigma$ to the $m$-fold symmetric bi-univalent class $\Sigma_{m}$. See [28] for further details regarding to this class $\Sigma_{m}$. Also, for coefficient problems of various subclasses of $\Sigma_{m}$ see [2, 25, 26, 30, 22] etc.

In order to prove our theorems, we need the following result.

Lemma 1.1. [15] Let the function $v \in \mathcal{P}$ be given by the following series:

$$
v(z)=1+v_{1} z+v_{2} z^{2}+\cdots, \quad(z \in \mathcal{U}) .
$$

The following sharp estimate holds true:

$$
\left|v_{n}\right| \leq 2 \quad(n \in \mathcal{N})
$$

If this $v(z)$ belongs to $\Sigma_{m}$ (see [16]), it takes the form

$$
v(z)=1+v_{m} z^{m}+v_{2 m} z^{2 m}+v_{3 m} z^{3 m}+\cdots, \quad(z \in \mathbb{U}) .
$$

In the present work, with reference to the Sakaguchi type function classes defined by Lokesh and Keerthi [11], we obtain estimates on initial coefficients

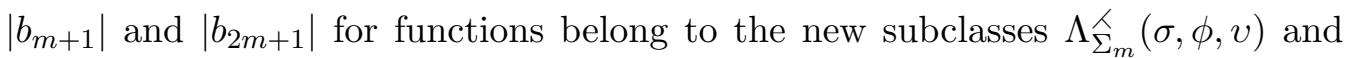
$\Lambda_{\Sigma_{m}}(\sigma, \gamma, v)$ of the function class $\Sigma_{m}$. Also, we have pointed out connections with certain familiar subclasses of the class $\Sigma$. 


\section{Coefficient Bounds for the Function Class}

$\Lambda_{\Sigma_{m}}^{\wedge}(\sigma, \phi, v)$

Definition 2.1. A function $p(z) \in \Sigma_{m}$ given by $(1.3)$ is said to be in the class $\Lambda_{\Sigma_{m}}(\sigma, \phi, v)$ if the following conditions are fulfilled:

$$
\begin{gathered}
\left|\arg \left[\frac{((1-\sigma) z)^{1-v}\left(p^{\prime}(z)\right)^{\wedge}}{(p(z)-p(\sigma z))^{1-v}}\right]\right|<\frac{\phi \pi}{2}, \\
(0<\phi \leq 1,0 \leq v<1,|\sigma| \leq 1, \curlywedge \geq 1 \text { but } \sigma \neq 1, z \in \mathcal{U})
\end{gathered}
$$

and

$$
\begin{gathered}
\left|\arg \left[\frac{((1-\sigma) w)^{1-v}\left(q^{\prime}(w)\right)^{\curlywedge}}{(q(w)-q(\sigma w))^{1-v}}\right]\right|<\frac{\phi \pi}{2}, \\
(0<\phi \leq 1,0 \leq v<1,|\sigma| \leq 1, \curlywedge \geq 1 \text { but } \sigma \neq 1, w \in \mathcal{U})
\end{gathered}
$$

where the function $q=p^{-1}$ is of the form 1.4 .

Theorem 2.2. Let $p$ given by 1.3 be in the class $\Lambda_{\widehat{\Sigma}_{m}}(\sigma, \phi, v), 0<\phi \leq 1$. Then

$$
\begin{aligned}
& \left|b_{m+1}\right| \leq \min \left\{\frac{2 \phi}{\lambda(m+1)-(1-v) \nu_{m+1}},\right.
\end{aligned}
$$

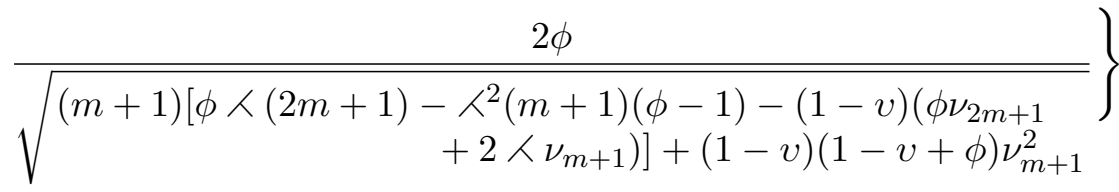

and

$$
\left|b_{2 m+1}\right| \leq \frac{2 \phi^{2}(m+1)}{\left[<(m+1)-(1-v) \nu_{m+1}\right]^{2}}+\frac{2 \phi}{\left[(2 m+1) \curlywedge-(1-v) \nu_{2 m+1}\right]},
$$

where $\nu_{m+1}=1+\sigma+\cdots+\sigma^{m}, \quad \nu_{2 m+1}=1+\sigma+\cdots+\sigma^{2 m}$.

Proof. Given $p \in \Lambda_{\Sigma_{m}}(\sigma, \phi, v)$. Thus,

$$
\left[\frac{((1-\sigma) z)^{1-v}\left(p^{\prime}(z)\right)^{\curlywedge}}{(p(z)-p(\sigma z))^{1-v}}\right]=[v(z)]^{\phi},
$$




$$
\left[\frac{((1-\sigma) w)^{1-v}\left(q^{\prime}(w)\right)^{\curlywedge}}{(q(w)-q(\sigma w))^{1-v}}\right]=[y(w)]^{\phi},
$$

where $v(z)$ and $y(w)$ in $\mathcal{P}$ with the series representation

$$
v(z)=1+v_{m} z^{m}+v_{2 m} z^{2 m}+\cdots, \quad y(z)=1+y_{m} z^{m}+y_{2 m} z^{2 m}+\cdots .
$$

Now, equating the coefficients in (2.5) and (2.6), we have

$$
\begin{gathered}
{\left[\curlywedge(m+1)-(1-v) \nu_{m+1}\right] b_{m+1}=\phi v_{m}} \\
{\left[\curlywedge(2 m+1)-(1-v) \nu_{2 m+1}\right] b_{2 m+1}+\frac{1}{2}\left[(1-v)\left((2-v) \nu_{m+1}-2 \curlywedge(m+1)\right)\right.} \\
\left.\nu_{m+1}+\curlywedge(\curlywedge-1)(m+1)^{2}\right] b_{m+1}^{2}=\phi v_{2 m}+\frac{\phi(\phi-1)}{2} v_{m}^{2}, \\
-\left[\curlywedge(m+1)-(1-v) \nu_{m+1}\right] b_{m+1}=\phi y_{m} \\
{\left[\curlywedge(2 m+1)-(1-v) \nu_{2 m+1}\right]\left[(m+1) b_{m+1}^{2}-b_{2 m+1}\right]+\frac{1}{2}\left[( 1 - v ) \left((2-v) \nu_{m+1}\right.\right.} \\
\left.-2 \curlywedge(m+1)) \nu_{m+1}+\curlywedge(\curlywedge-1)(m+1)^{2}\right] b_{m+1}^{2}=\phi y_{2 m}+\frac{\phi(\phi-1)}{2} y_{m}^{2} .
\end{gathered}
$$

From (2.7) and $(2.9)$ we obtain

$$
\begin{gathered}
v_{m}=-y_{m}, \\
2\left[\wedge(m+1)-(1-v) \nu_{m+1}\right]^{2} b_{m+1}^{2}=\phi^{2}\left[v_{m}^{2}+y_{m}^{2}\right] .
\end{gathered}
$$

Also from (2.8), (2.10) and (2.12) we have

$$
\begin{gathered}
\left\{(m+1)\left[\curlywedge(2 m+1)-(1-v) \nu_{2 m+1}\right]+(1-v) \nu_{m+1}\left((2-v) \nu_{m+1}-2 \curlywedge(m+1)\right)\right. \\
\left.+\wedge(\curlywedge-1)(m+1)^{2}\right\} b_{m+1}^{2}=\phi\left(v_{2 m}+y_{2 m}\right) \\
+\frac{\phi(\phi-1)\left[\curlywedge(m+1)-(1-v) \nu_{m+1}\right]^{2}}{\phi^{2}} b_{m+1}^{2}
\end{gathered}
$$


Therefore, we have

$$
b_{m+1}^{2}=\frac{\phi^{2}\left(v_{2 m}+y_{2 m}\right)}{(m+1)\left[\phi \curlywedge(2 m+1)-\curlywedge^{2}(m+1)(\phi-1)-(1-v)\right.} .
$$

Thus, we obtain from relations 2.12 and 2.14 that

$$
\left|b_{m+1}^{2}\right| \leq \frac{\phi^{2}\left[\left|v_{m}^{2}\right|+\left|y_{m}^{2}\right|\right]}{2\left[\wedge(m+1)-(1-v) \nu_{m+1}\right]^{2}}
$$

and

$$
\left|b_{m+1}^{2}\right| \leq \frac{\phi^{2}\left(\left|v_{2 m}\right|+\left|y_{2 m}\right|\right)}{(m+1)\left[\phi \curlywedge(2 m+1)-\curlywedge^{2}(m+1)(\phi-1)-(1-v)\right.},
$$

respectively. Applying Lemma 1.1 for the coefficients $v_{m}, v_{2 m}, y_{m}, y_{2 m}$ we have the desired estimates on $\left|b_{m+1}\right|$ as given in $(2.3)$. Next, in order to find the bound on $\left|b_{2 m+1}\right|$, by subtracting 2.10 from 2.8, we obtain

$$
\begin{array}{r}
2\left[\wedge(2 m+1)-(1-v) \nu_{2 m+1}\right] b_{2 m+1}-\left[(2 m+1) \nless-(1-v) \nu_{2 m+1}\right](m+1) b_{m+1}^{2} \\
=\phi\left[v_{2 m}-y_{2 m}\right]+\frac{\phi(\phi-1)}{2}\left[v_{m}^{2}-y_{m}^{2}\right] .
\end{array}
$$

Then, in view of 2.11) and (2.12), and applying Lemma 1.1 for the coefficients $v_{m}, v_{2 m}, y_{m}, y_{2 m}$, we have

$$
\left|b_{2 m+1}\right| \leq \frac{2 \phi^{2}(m+1)}{\left[\curlywedge(m+1)-(1-v) \nu_{m+1}\right]^{2}}+\frac{2 \phi}{\left[(2 m+1) \curlywedge-(1-v) \nu_{2 m+1}\right]} .
$$

This completes the proof of Theorem 2.2 .

\section{Coefficient Bounds for the Function Class $\Lambda_{\Sigma_{m}}(\sigma, \gamma, v)$}

Definition 3.1. A function $p(z) \in \Sigma_{m}$ given by 1.3 is said to be in the class $\Lambda_{\Sigma_{m}}(\sigma, \gamma, v)$ if the following conditions are fulfilled:

$$
\left[\frac{((1-\sigma) z)^{1-v}\left(p^{\prime}(z)\right)^{\wedge}}{(p(z)-p(\sigma z))^{1-v}}\right]>\gamma
$$




$$
(0 \leq \gamma<1,0 \leq v<1,|\sigma| \leq 1, \wedge \geq 1 \text { but } \sigma \neq 1, z \in \mathcal{U})
$$

and

$$
\begin{gathered}
{\left[\frac{((1-\sigma) w)^{1-v}\left(q^{\prime}(w)\right)^{\curlywedge}}{(q(w)-q(\sigma w))^{1-v}}\right]>\gamma,} \\
(0 \leq \gamma<1,0 \leq v<1,|\sigma| \leq 1, \wedge \geq 1 \text { but } \sigma \neq 1, w \in \mathcal{U})
\end{gathered}
$$

where the function $q=p^{-1}$ is of the form (1.4).

Theorem 3.2. Let $p(z)$ given by 1.3 be in the class $\Lambda_{\Sigma_{m}}(\sigma, \gamma, v), 0 \leq \gamma<1$. Then

$$
\begin{aligned}
\left|b_{m+1}\right| & \leq \min \left\{\frac{2(1-\gamma)}{\lambda(m+1)-(1-v) \nu_{m+1}},\right. \\
& \left.\frac{2 \sqrt{1-\gamma}}{\sqrt{(m+1)\left[\curlywedge(2 m+1)-(1-v) \nu_{2 m+1}\right]+(1-v)\left((2-v) \nu_{m+1}\right.}}\right\}
\end{aligned}
$$

and

$$
\left|b_{2 m+1}\right| \leq \frac{2(1-\gamma)^{2}(m+1)}{\left[\measuredangle(m+1)-(1-v) \nu_{m+1}\right]^{2}}+\frac{2(1-\gamma)}{(2 m+1) \curlywedge-(1-v) \nu_{2 m+1}},
$$

where $\nu_{m+1}=1+\sigma+\cdots+\sigma^{m}, \quad \nu_{2 m+1}=1+\sigma+\cdots+\sigma^{2 m}$.

Proof. Given $p \in \Lambda_{\Sigma_{m}}^{\wedge}(\sigma, \gamma, v)$. Thus,

$$
\begin{gathered}
{\left[\frac{((1-\sigma) z)^{1-v}\left(p^{\prime}(z)\right)^{\curlywedge}}{(p(z)-p(\sigma z))^{1-v}}\right]=\gamma+(1-\gamma) v(z),} \\
{\left[\frac{((1-\sigma) w)^{1-v}\left(q^{\prime}(w)\right)^{\curlywedge}}{(q(w)-q(\sigma w))^{1-v}}\right]=\gamma+(1-\gamma) y(w),}
\end{gathered}
$$

where $v, y \in \mathcal{P}$ and $q=p^{-1}$.

It follows from (3.5) and (3.6) that

$$
\left[\curlywedge(m+1)-(1-v) \nu_{m+1}\right] b_{m+1}=(1-\gamma) v_{m},
$$




$$
\begin{aligned}
& {\left[\curlywedge(2 m+1)-(1-v) \nu_{2 m+1}\right] b_{2 m+1}+\frac{1}{2}\left[(1-v)\left((2-v) \nu_{m+1}-2 \curlywedge(m+1)\right)\right.} \\
& \left.\nu_{m+1}+\curlywedge(\curlywedge-1)(m+1)^{2}\right] b_{m+1}^{2}=(1-\gamma) v_{2 m} \\
& -\left[\wedge(m+1)-(1-v) \nu_{m+1}\right] b_{m+1}=(1-\gamma) y_{m}, \\
& {\left[\wedge(2 m+1)-(1-v) \nu_{2 m+1}\right]\left[(m+1) b_{m+1}^{2}-b_{2 m+1}\right]+\frac{1}{2}\left[( 1 - v ) \left((2-v) \nu_{m+1}\right.\right.} \\
& \left.-2 \curlywedge(m+1)) \nu_{m+1}+\measuredangle(\curlywedge-1)(m+1)^{2}\right] b_{m+1}^{2}=(1-\gamma) y_{2 m} .
\end{aligned}
$$

From 3.7 and (3.9) we obtain

$$
\begin{gathered}
v_{m}=-y_{m} \\
2\left[\wedge(m+1)-(1-v) \nu_{m+1}\right]^{2} b_{m+1}^{2}=(1-\gamma)^{2}\left(v_{m}^{2}+y_{m}^{2}\right) .
\end{gathered}
$$

Also from 3.8, 3.10 and 3.12 we have

$$
\begin{aligned}
\{(m+1)[\curlywedge(2 m+1)- & \left.(1-v) \nu_{2 m+1}\right]+(1-v) \nu_{m+1}\left((2-v) \nu_{m+1}-2 \curlywedge(m+1)\right) \\
& \left.+\curlywedge(\wedge-1)(m+1)^{2}\right\} b_{m+1}^{2}=(1-\gamma)\left(v_{2 m}+y_{2 m}\right) .
\end{aligned}
$$

Therefore, we have

$$
b_{m+1}^{2}=\frac{(1-\gamma)\left(v_{2 m}+y_{2 m}\right)}{(m+1)\left[\curlywedge(2 m+1)-(1-v) \nu_{2 m+1}\right]+(1-v) \nu_{m+1}\left((2-v) \nu_{m+1}\right.} .
$$

Thus, we obtain from relations 3.12 and 3.14 that

$$
\left|b_{m+1}^{2}\right| \leq \frac{(1-\gamma)^{2}\left[\left|v_{m}^{2}\right|+\left|y_{m}^{2}\right|\right]}{2\left[\wedge(m+1)-(1-v) \nu_{m+1}\right]^{2}}
$$


and

$$
\begin{array}{r}
\left|b_{m+1}^{2}\right| \leq \frac{(1-\gamma)\left(\left|v_{2 m}\right|+\left|y_{2 m}\right|\right)}{(m+1)\left[\curlywedge(2 m+1)-(1-v) \nu_{2 m+1}\right]+(1-v) \nu_{m+1}\left((2-v) \nu_{m+1}\right.} \\
-2 \curlywedge(m+1))+\curlywedge(\ltimes-1)(m+1)^{2}
\end{array}
$$

respectively. Applying Lemma 1.1 for the coefficients $v_{m}, v_{2 m}, y_{m}, y_{2 m}$ we have the desired estimates on $\left|b_{m+1}\right|$ as given in (3.3). Next, in order to find the bound on $\left|b_{2 m+1}\right|$, by subtracting (3.10) from 3.8, we obtain

$$
\begin{aligned}
2\left[\wedge(2 m+1)-(1-v) \nu_{2 m+1}\right] b_{2 m+1}-[(2 m+1) & \left.\wedge-(1-v) \nu_{2 m+1}\right](m+1) b_{m+1}^{2} \\
= & (1-\gamma)\left(v_{2 m}-y_{2 m}\right) .
\end{aligned}
$$

Then, in view of (3.11) and (3.12), and applying Lemma 1 for the coefficients $v_{m}, v_{2 m}, y_{m}, y_{2 m}$, we have

$$
\left|b_{2 m+1}\right| \leq \frac{2(1-\gamma)^{2}(m+1)}{\left[\measuredangle(m+1)-(1-v) \nu_{m+1}\right]^{2}}+\frac{2(1-\gamma)}{(2 m+1) \nless-(1-v) \nu_{2 m+1}} .
$$

This completes the proof of Theorem 3.2 .

\section{Corollaries and Consequences}

By setting $v=0$ in Theorems 2.2 and 3.2 , we have the following corollary.

Corollary 4.1. Let $p$ given by $(1.3)$ be in the class $\Lambda_{\Sigma_{m}}^{\wedge}(\sigma, \phi), 0<\phi \leq 1$. Then

$$
\begin{aligned}
& \left|b_{m+1}\right| \leq \min \left\{\frac{2 \phi}{\lambda(m+1)-\nu_{m+1}}\right.
\end{aligned}
$$

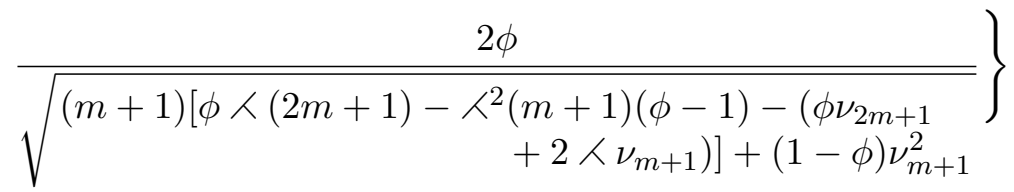

and

$$
\left|b_{2 m+1}\right| \leq \frac{2 \phi^{2}(m+1)}{\left[\wedge(m+1)-\nu_{m+1}\right]^{2}}+\frac{2 \phi}{(2 m+1) \nless-\nu_{2 m+1}},
$$

where $\nu_{m+1}=1+\sigma+\cdots+\sigma^{m}, \quad \nu_{2 m+1}=1+\sigma+\cdots+\sigma^{2 m}$. 


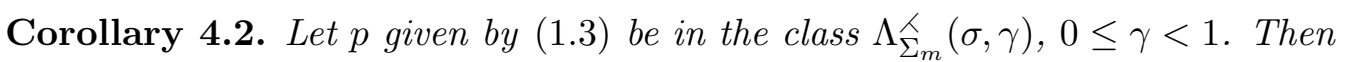

$$
\begin{aligned}
& \left|b_{m+1}\right| \leq \min \left\{\frac{2(1-\gamma)}{\lambda(m+1)-\nu_{m+1}},\right.
\end{aligned}
$$

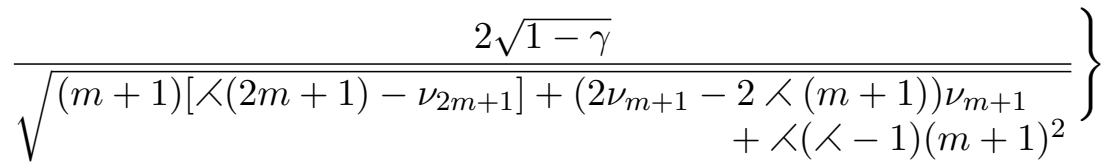

and

$$
\left|b_{2 m+1}\right| \leq \frac{2(1-\gamma)^{2}(m+1)}{\left[\measuredangle(m+1)-\nu_{m+1}\right]^{2}}+\frac{2(1-\gamma)}{(2 m+1) \curlywedge-\nu_{2 m+1}},
$$

where $\nu_{m+1}=1+\sigma+\cdots+\sigma^{m}, \nu_{2 m+1}=1+\sigma+\cdots+\sigma^{2 m}$.

By setting $m=1$ (1-fold) in Corollaries 4.1 and 4.2 , we have the Corollaries 4.3 and 4.4 as follows:

Corollary 4.3. Let $f$ given by (1.1) be in the class $\Lambda_{\Sigma}^{\widehat{\Sigma}}(\sigma, \phi), 0<\phi \leq 1$. Then

$$
\begin{aligned}
& \left|a_{2}\right| \leq \min \left\{\frac{2 \phi}{2 \curlywedge-(1+\sigma)},\right.
\end{aligned}
$$

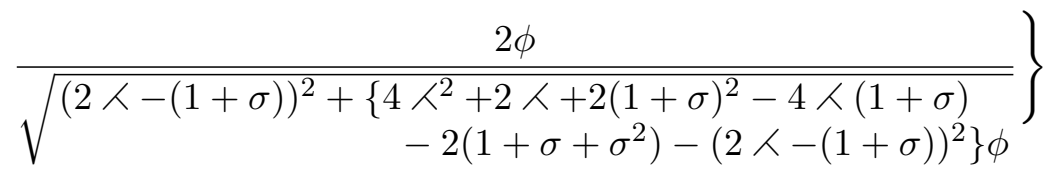

and

$$
\left|a_{3}\right| \leq \frac{4 \phi^{2}}{[2 \curlywedge-(1+\sigma)]^{2}}+\frac{2 \phi}{3 \curlywedge-\left(1+\sigma+\sigma^{2}\right)} .
$$

Corollary 4.4. Let $f$ given by (1.1) be in the class $\Lambda_{\Sigma}^{\wedge}(\sigma, \gamma), 0 \leq \gamma<1$. Then

$$
\begin{aligned}
\left|a_{2}\right| \leq \min \left\{\frac{2(1-\gamma)}{2 \curlywedge-(1+\sigma)},\right. & \\
& \left.\frac{2 \sqrt{1-\gamma}}{\sqrt{\left(4 \curlywedge^{2}+2 \curlywedge-4 \curlywedge(1+\sigma)+2(1+\sigma)^{2}-2\left(1+\sigma+\sigma^{2}\right)\right)}}\right\}
\end{aligned}
$$

and

$$
\left|a_{3}\right| \leq \frac{4(1-\gamma)^{2}}{[2 \curlywedge-(1+\sigma)]^{2}}+\frac{2(1-\gamma)}{3 \curlywedge-\left(1+\sigma+\sigma^{2}\right)} .
$$


Remark 4.5. The above estimates $\left|a_{2}\right|$ and $\left|a_{3}\right|$ show that Corollaries 4.3 and 4.4 is an improvement of the estimate obtain by Lokesh and Keerthi ([11], Corollaries 3.1 and 3.2).

Corollary 4.6. [11] Let $f$ given by [1.1] be in the class $\Lambda_{\Sigma}(\sigma, \phi), 0<\phi \leq 1$. Then

$$
\left|a_{2}\right| \leq \frac{2 \phi}{\sqrt{(2 \curlywedge-(1+\sigma))^{2}+\left\{4 \curlywedge^{2}+2 \curlywedge+2(1+\sigma)^{2}-4 \curlywedge(1+\sigma)\right.}}
$$

and

$$
\left|a_{3}\right| \leq \frac{4 \phi^{2}}{[2 \curlywedge-(1+\sigma)]^{2}}+\frac{2 \phi}{3 \curlywedge-\left(1+\sigma+\sigma^{2}\right)} .
$$

Corollary 4.7. [11] Let $f$ given by [1.1] be in the class $\Lambda_{\Sigma}(\sigma, \gamma), 0 \leq \gamma<1$. Then

$$
\left|a_{2}\right| \leq \frac{2 \sqrt{1-\gamma}}{\sqrt{\left(4 \curlywedge^{2}+2 \curlywedge-4 \curlywedge(1+\sigma)+2(1+\sigma)^{2}-2\left(1+\sigma+\sigma^{2}\right)\right)}}
$$

and

$$
\left|a_{3}\right| \leq \frac{4(1-\gamma)^{2}}{[2 \curlywedge-(1+\sigma)]^{2}}+\frac{2(1-\gamma)}{3 \curlywedge-\left(1+\sigma+\sigma^{2}\right)} .
$$

By setting $\curlywedge=0$ in Theorems 2.2 and 3.2 , we have the following corollary.

Corollary 4.8. Let $p$ given by (1.3) be in the class $\Lambda_{\Sigma_{m}}(\sigma, \phi, v), 0<\phi \leq 1$. Then

$$
\begin{aligned}
& \left|b_{m+1}\right| \leq \min \left\{\frac{2 \phi}{(m+1)-(1-v) \nu_{m+1}},\right.
\end{aligned}
$$

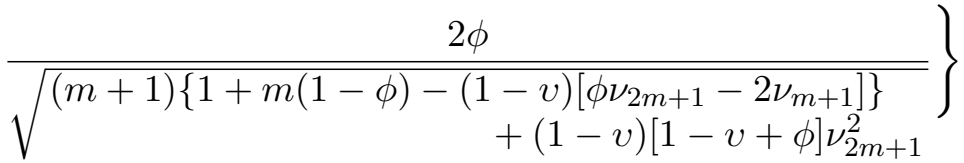

and

$$
\left|b_{2 m+1}\right| \leq \frac{2 \phi^{2}(m+1)}{\left[(m+1)-(1-v) \nu_{m+1}\right]^{2}}+\frac{2 \phi}{\left[(2 m+1)-(1-v) \nu_{2 m+1}\right]},
$$

where $\nu_{m+1}=1+\sigma+\cdots+\sigma^{m}, \quad \nu_{2 m+1}=1+\sigma+\cdots+\sigma^{2 m}$. 
Corollary 4.9. Let $p$ given by $(1.3)$ be in the class $\Lambda_{\Sigma_{m}}(\sigma, \gamma, v), 0 \leq \gamma<1$. Then

$$
\begin{aligned}
\left|b_{m+1}\right| \leq \min & \left\{\frac{2(1-\gamma)}{(m+1)-(1-v) \nu_{m+1}},\right. \\
& \left.\frac{2 \sqrt{1-\gamma}}{\sqrt{(m+1)\left[(2 m+1)-(1-v) \nu_{2 m+1}\right]+(1-v)\left((2-v) \nu_{m+1}\right.}}\right\}
\end{aligned}
$$

and

$$
\left|b_{2 m+1}\right| \leq \frac{2(1-\gamma)^{2}(m+1)}{\left[(m+1)-(1-v) \nu_{m+1}\right]^{2}}+\frac{2(1-\gamma)}{(2 m+1)-(1-v) \nu_{2 m+1}},
$$

where $\nu_{m+1}=1+\sigma+\cdots+\sigma^{m}, \quad \nu_{2 m+1}=1+\sigma+\cdots+\sigma^{2 m}$.

Remark 4.10. The above estimates $\left|b_{m+1}\right|$ and $\left|b_{2 m+1}\right|$ show that Corollaries 4.8 and 4.9 is an improvement of the estimate obtain by Senthil and Keerthi ([18], Theorem 6 and 7).

Corollary 4.11. [18] Let $p$ given by [1.3] be in the class $\Lambda_{\Sigma_{m}}(\sigma, \phi, v), 0<\phi \leq 1$. Then

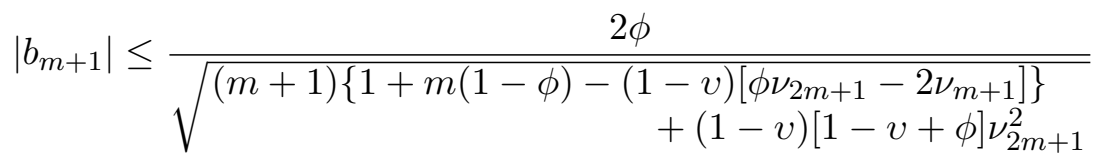

and

$$
\left|b_{2 m+1}\right| \leq \frac{2 \phi^{2}(m+1)}{\left[(m+1)-(1-v) \nu_{m+1}\right]^{2}}+\frac{2 \phi}{\left[(2 m+1)-(1-v) \nu_{2 m+1}\right]},
$$

where $\nu_{m+1}=1+\sigma+\cdots+\sigma^{m}, \nu_{2 m+1}=1+\sigma+\cdots+\sigma^{2 m}$.

Corollary 4.12. [18] Let $p$ given by [1.3] be in the class $\Lambda_{\Sigma_{m}}(\sigma, \gamma, v), 0 \leq \gamma<1$. Then

$$
\left|b_{m+1}\right| \leq \frac{2 \sqrt{1-\gamma}}{\sqrt{(m+1)\left[(2 m+1)-(1-v) \nu_{2 m+1}\right]+(1-v)\left((2-v) \nu_{m+1}\right.}}
$$

and

$$
\left|b_{2 m+1}\right| \leq \frac{2(1-\gamma)^{2}(m+1)}{\left[(m+1)-(1-v) \nu_{m+1}\right]^{2}}+\frac{2(1-\gamma)}{(2 m+1)-(1-v) \nu_{2 m+1}},
$$

where $\nu_{m+1}=1+\sigma+\cdots+\sigma^{m}, \nu_{2 m+1}=1+\sigma+\cdots+\sigma^{2 m}$. 


\section{References}

[1] R. M. Ali, S. K. Lee, V. Ravichandran and S. Supramaniam, Coefficient estimates for bi-univalent Ma-Minda starlike and convex functions, Appl. Math. Lett. 25 (2012), 344-351. https://doi.org/10.1016/j.aml.2011.09.012

[2] S. Altinkaya and S. Yalçin, On some subclasses of $m$-fold symmetric bi-univalent functions, 2018. arXiv:1603.01120

[3] D. A. Brannan and J. G. Clunie, Aspects of Contemporary Complex Analysis, Academic Press, London, 1980.

[4] D. A. Brannan and T. S. Taha, On some classes of bi-univalent functions, Stud. Univ. Babeş-Bolyai Math. 31(2) (1986), 70-77.

[5] P. L. Duren, Univalent Functions, Grundlehren der Mathematischen Wissenschaften, Springer, New York, 1983.

[6] B. A. Frasin and M. K. Aouf, New subclasses of bi-univalent functions, Appl. Math. Lett. 24 (2011), 1569-1573. https://doi.org/10.1016/j.aml.2011.03.048

[7] A. W. Goodman, An invitation on the study of univalent and multivalent functions, Int. J. Math. Math. Sci. 2 (1979), Article ID 306560, 24 pp. https://doi.org/10.1155/S016117127900017X

[8] S. Joshi, S. Joshi and H. Pawar, On some subclasses of bi-univalent functions associated with pseudo-starlike functions, J. Egyptian Math. Soc. 24 (2016), 522-525. https://doi.org/10.1016/j.joems.2016.03.007

[9] W. Koepf, Coefficients of symmetric functions of bounded boundary rotations, Proc. Amer. Math. Soc. 105 (1989), 324-329.

https://doi.org/10.1090/S0002-9939-1989-0930244-7

[10] M. Lewin, On a coefficient problem for bi-univalent functions, Proc. Amer. Math. Soc. 18 (1967), 63-68. https://doi.org/10.1090/S0002-9939-1967-0206255-1

[11] P. Lokesh and B. S. Keerthi, Results on Bazilevic Sakaguchi type of functions, Adv. Math., Sci. J. 9(8) (2020), 5763-5774.

https://doi.org/10.37418/amsj.9.8.44 
[12] E. Netanyahu, The minimal distance of the image boundary from the origin and the second coefficient of a univalent function in $|z|<1$, Arch. Ration. Mech. Anal. 32 (1969), 100-112. https://doi.org/10.1007/BF00247676

[13] A. B. Patil and U. H. Naik, Bounds on initial coefficients for a new subclass of bi-univalent functions, New Trends Math. Sci. 6(1) (2018), 85-90. https://doi.org/10.20852/ntmsci.2018.248

[14] A. B. Patil and T. G. Shaba, On sharp Chebyshev polynomial bounds for general subclass of bi-univalent functions, Applied Sciences 23 (2021), 109-117.

[15] Ch. Pommerenke, Univalent Functions, Vandenhoeck and Rupercht, Göttingen, 1975.

[16] Ch. Pommerenke, On the coefficients of close-to-convex functions, Michigan Math. J. 9 (1962), 259-269. https://doi.org/10.1307/mmj/1028998726

[17] S. Porwal and M. Darus, On a new subclass of bi-univalent functions, J. Egyptian Math. Soc. 21(3) (2013), 190-193. https://doi.org/10.1016/j.joems.2013.02.007

[18] B. Senthil and B. S. Keerthi, Certain subclasses of $m$-fold symmetric-Sakaguchi type bi-univalent functions, Int. J. Pure Appl. Math. 109(10) (2016), 29-37.

[19] T. G. Shaba, On some new subclass of bi-univalent functions associated with the Opoola differential operator, Open J. Math. Anal. 4(2) (2020), 74-79. https://doi.org/10.30538/psrp-oma2020.0064

[20] T. G. Shaba, On some subclasses of bi-pseudo-starlike functions defined by Salagean differential operator, Asia Pac. J. Math. 8(6) (2021), 1-11.

https://doi.org/10.28924/apjm/8-6

[21] T. G. Shaba, M. G. Khan and B. Ahmed, Coefficient bounds for certain subclasses of meromorphic bi-univalent functions associated with Al-Oboudi differential operator, Palestine J. Math., accepted.

[22] T. G. Shaba and A. B. Patil, Coefficient estimates for certain subclasses of $m$-fold symmetric bi-univalent functions associated with pseudo-starlike functions, Earthline J. Math. Sci. 6(2) (2021) 209-223.

https://doi.org/10.34198/ejms.6221.209223 
[23] T. G. Shaba and A. K. Wanas, Coefficient bounds for a new family of bi-univalent functions associated with $(U, V)$-Lucas polynomials, Int. J. Nonlinear Anal. Appl. 13(1) (2022), 615-626. https://dx.doi.org/10.22075/ijnaa.2021.23927.2639

[24] H. M. Srivastava and D. Bansal, Coefficient estimates for a subclass of analytic and bi-univalent functions, J. Egyptian Math. Soc. 23(2) (2015), 242-246. https://doi.org/10.1016/j.joems.2014.04.002

[25] H. M. Srivastava, S. Gaboury and F. Ghanim, Coefficient estimates for some subclasses of $m$-fold symmetric bi-univalent functions, Acta Univ. Apulensis Math. 41 (2015), 153-164. https://doi.org/10.17114/j.aua.2015.41.12

[26] H. M. Srivastava, S. Gaboury and F. Ghanim, Initial coefficient estimates for some subclasses of $m$-fold symmetric bi-univalent functions, Acta Math. Sci. Ser. B Engl. Ed. 36(3) (2016), 863-871. https://doi.org/10.1016/S0252-9602(16)30045-5

[27] H. M. Srivastava, A. K. Mishra and P. Gochhayat, Certain subclasses of analytic and bi-univalent functions, Appl. Math. Lett. 23 (2010), 1188-1192. https://doi.org/10.1016/j.aml.2010.05.009

[28] H. M. Srivastava, S. Sivasubramanian and R. Sivakumar, Initial coefficient bounds for a subclass of $m$-fold symmetric bi-univalent functions, Tbilisi Math. J. 7(2) (2014), 1-10. https://doi.org/10.2478/tmj-2014-0011

[29] D. Styer and D. J. Wright, Results on bi-univalent functions, Proc. Amer. Math. Soc. 82(2) (1981), 243-248. https://doi.org/10.1090/S0002-9939-1981-0609659-5

[30] H. Tang, H. M. Srivastava, S. Sivasubramanian and P. Gurusamy, The Fekete-Szegö functional problems for some subclasses of $m$-fold symmetric bi-univalent functions, J. Mathcal. Inequalities 10(4) (2016), 1063-1092. https://doi.org/10.7153/jmi-10-85

This is an open access article distributed under the terms of the Creative Commons Attribution License (http://creativecommons.org/licenses/by/4.0/), which permits unrestricted, use, distribution and reproduction in any medium, or format for any purpose, even commercially provided the work is properly cited. 